\title{
The Vietoris system in strong shape and strong homology
}

\author{
by
}

\author{
Bernd G ünther (Frankfurt)
}

\begin{abstract}
We show that the Vietoris system of a space is isomorphic to a strong expansion of that space in the Steenrod homotopy category, and from this we derive a simple description of strong homology. It is proved that in ZFC strong homology does not have compact supports, and that enforcing compact supports by taking limits leads to a homology functor that does not factor over the strong shape category. For compact Hausdorff spaces strong homology is proved to be isomorphic to Massey's homology.
\end{abstract}

0. Introduction. In 1952 C. H. Dowker [5] proved that the cohomology groups of a topological space based on the Čech and the Vietoris constructions are isomorphic. However, since the Čech groups work very well in the context of cohomology, the Vietoris approach was abandoned until 1973 when it was taken up by Porter [23, 24, 25] and three years later by Edwards and Hastings [6, p. 250]. The last two authors observed that only the Vietoris system has the rigidity properties necessary for the construction of exact (generalized) homology theories for compact metric spaces with properties similar to Steenrod-Sitnikov homology. In case of ordinary homology the domain of definition was extended to arbitrary topological spaces by Miminoshvili in [22] and independently by Lisica and Mardešić in [11, 12, 13], using resolutions instead of one of the two classical systems; the resulting theory is known as strong homology. A similar development went on in shape theory: The Čech system served its purpose in ordinary shape theory $[18$, p. 49$]$ but had to be replaced by resolutions in strong shape theory $[4$, 10]. The disadvantage of resolutions is their lack of naturality.

1991 Mathematics Subject Classification: Primary 55P55, 55U15, 55N07; Secondary $54 \mathrm{E} 35,55 \mathrm{U} 35$

Key words and phrases: Vietoris nerve, Steenrod homotopy category, strong shape theory, strong homology, compact supports.

This article was written while the author was visiting the University of Washington, Seattle, supported by a DFG fellowship. He acknowledges inspiring conversations with J. Segal, E. Michael, S. Spież and R. Pol. 
In Section 1 of this paper we are going to prove that resolutions may be replaced by the Vietoris system in the context of strong shape theory. This will allow us to give a rather nice and simple presentation of strong homology, making this subject accessible for non-specialists in strong shape theory. We describe this form of homology in Section 2 and give self-contained proofs of the Eilenberg-Steenrod axioms. For compact Hausdorff spaces this approach is close to Massey's homology, and we give a proof of isomorphism in Section 3. There we also consider strong homology with compact supports. It has some advantages over the usual form of strong homology (it occurs in Sitnikov's duality theorem, and the question whether the homology functors in negative dimensions are trivial is decidable), but also some disadvantages (most notably: it does not factor over the strong shape category). We show that strong homology in its usual form does not have compact supports (in ZFC).

1. The Vietoris system. We consider the set $M_{X}$ of all continuous pseudometrics (hereafter simply called metrics) on a given topological space $X ; M_{X}$ is directed if we define $d \leq d^{\prime} \Leftrightarrow \forall x, y \in X: d(x, y) \leq d^{\prime}(x, y)$. The Vietoris nerve of a metric $d$ is the simplicial complex whose $n$-simplices are the finite sets $A \subseteq X$ with $d$-diameter $\delta_{d}(A)$ at most 1 . By $N(d)$ we denote the Vietoris nerve with the metric topology [18, p. 301]. When $d$ varies in $M_{X}$ the polyhedra $N(d)$ form an inverse system $\mathbf{V}(X)$ with inclusions as bonding maps, which is called the Vietoris system of $X$. The Vietoris system has the interesting property of depending strictly functorially on $X$ : Every continuous map $f: X \rightarrow Y$ induces a morphism $\mathbf{V}(f): \mathbf{V}(X) \rightarrow \mathbf{V}(Y)$ in pro-Top (even more: in inv-Top) composed of the nondecreasing index map $f^{*}: M_{Y} \rightarrow M_{X}, f^{*} d\left(x, x^{\prime}\right):=d\left(f(x), f\left(x^{\prime}\right)\right)$, and the simplicial maps $f_{*}: N\left(f^{*} d\right) \rightarrow N(d)$ mapping every vertex $x$ onto $f(x)$. This property distinguishes the Vietoris system from related constructions working only on the homotopy level (such as the Čech system) or being natural only up to coherent homotopy (such as resolutions), and makes it particularly useful for strong shape theory. There one needs to assign a strong ANR-expansion [15, Def. 1] to every space to obtain a functor $\mathbf{L}: \mathbf{H T o p} \rightarrow \mathbf{H}$ (pro-Top) into the Steenrod homotopy category [4, Def. 1.11]; the strong shape category ssh is then defined by $\mathrm{Ob}(\mathbf{s s h}):=\mathrm{Ob}(\mathbf{H T o p})$ and $\operatorname{ssh}(X, Y):=$ $\mathbf{H}$ (pro-Top) $(\mathbf{L}(X), \mathbf{L}(Y))$. A strong ANR-expansion of a space $X$ consists of an inverse system of ANR-spaces $\mathbf{L}(X)=\left\{f_{\lambda}^{\mu}: P_{\mu} \rightarrow P_{\lambda} \mid \mu \geq \lambda \in \Lambda\right\}$ and a morphism $\left\{g_{\lambda}\right\}: X \rightarrow \mathbf{L}(X) \in$ pro-Top satisfying the following two conditions:

(a) Any map $h: X \rightarrow Y$ into an ANR-space $Y$ factors over some $g_{\lambda}$ up to homotopy.

(b) For any two maps $h_{0}, h_{1}: P_{\lambda} \rightarrow Y$ into an ANR-space and any 
homotopy $H: h_{0} g_{\lambda} \simeq h_{1} g_{\lambda}$ there exist an index $\mu \geq \lambda$ and a homotopy $G: h_{0} f_{\lambda}^{\mu} \simeq h_{1} f_{\lambda}^{\mu}$ with $G\left(g_{\mu} \times \mathrm{id}\right) \simeq H$ relative $X \times \dot{I}$.

The main result of this section is to show that $\mathbf{L}(X)$ is isomorphic to the Vietoris system $\mathbf{V}(X)$ in the Steenrod homotopy category.

Remark. The original construction of the Vietoris system in [23] used open coverings $\mathcal{U}$ of $X$ instead of metrics as index set and specified the simplices of $N(\mathcal{U})$ to be the $\mathcal{U}$-small, finite subsets of $X$. Using the fact that each open covering $\mathcal{U}$ of a paracompact space $X$ admits a metric $d$ such that the open balls of radius 1 with respect to $d$ are $\mathcal{U}$-small the reader may convince himself that in the paracompact case the two constructions are equivalent. For general spaces open coverings have to be replaced by normal ones.

Definition 1. Let $d$ be a metric on a space $X$ and $N(d)$ the Vietoris nerve of $d$. A map $q: X \rightarrow N(d)$ is barycentric if $\delta_{d}\left(\{x\} \cup q^{-1}(\right.$ st $\left.x)\right) \leq 1 / 3$ for each $x \in X$.

In the formula above the letter $x$ denotes once a point of $X$ and secondly the corresponding vertex of $N(d)$.

Proposition 1. Every metric $d$ on an arbitrary space $X$ admits a barycentric map $q: X \rightarrow N(d)$. If $q_{0}, \ldots, q_{n}: X \rightarrow N(d)$ is a finite collection of barycentric maps, then for fixed $x \in X$ the points $q_{0}(x), \ldots, q_{n}(x) \in N(d)$ lie in a single simplex, so that we can define a map $\Gamma: X \times \Delta^{n} \rightarrow N(d)$ by $\Gamma\left(x, t_{0}, \ldots, t_{n}\right):=\sum_{k=0}^{n} t_{k} q_{k}(x)$.

Proof. To construct a barycentric map $q: X \rightarrow N(d)$ we take a locally finite partition of unity $\left\{\varphi_{\iota} \mid \iota \in J\right\}$ on $X$ with $\left.\left.\delta_{d}\left(\varphi_{\iota}^{-1}\right] 0,1\right]\right) \leq 1 / 6$ for every index $\iota$ and choose $x_{\iota} \in X$ with $\varphi_{\iota}\left(x_{\iota}\right)>0$. For $x \in X$ we denote by $J_{x} \subseteq J$ the finite set of all indices $\iota$ with $\varphi_{\iota}(x)>0$. If $\iota$ and $\kappa$ belong to $J_{x}$ we have $d\left(x, x_{\iota}\right), d\left(x, x_{\kappa}\right) \leq 1 / 6$ and therefore $d\left(x_{\iota}, x_{\kappa}\right) \leq 1 / 3 \leq 1$. Hence $\left\{x_{\iota} \mid \iota \in J_{x}\right\}$ spans a simplex of $N(d)$ and we can define

$$
q(x):=\sum_{\iota} \varphi_{\iota}(x) x_{\iota} .
$$

We claim that this map $q$ is barycentric. If $q(y)$ belongs to st $x$, then there must be an index $\iota$ with $x_{\iota}=x$ and $\varphi_{\iota}(y)>0$. By construction we have $\varphi_{\iota}\left(x_{\iota}\right)>0$ and therefore $d(y, x) \leq 1 / 6$. If $y^{\prime}$ is another point of $q^{-1}$ (st $x$ ) then $d\left(y, y^{\prime}\right) \leq d(y, x)+d\left(y^{\prime}, x\right) \leq 1 / 3$ and therefore the map $q$ is barycentric.

The second part of Proposition 1 follows from Lemma 1, applied to $x_{0}=\ldots=x_{n}:=x$ :

LEMma 1. If $q_{0}, \ldots, q_{n}: X \rightarrow N(d)$ is a finite collection of barycentric maps and if $\left\{x_{0}, \ldots, x_{n}\right\} \subseteq X$ has diameter at most $1 / 3$, then there is 
a simplex of $N(d)$ containing the vertices $x_{0}, \ldots, x_{n}$ as well as the points $q_{0}\left(x_{0}\right), \ldots, q_{n}\left(x_{n}\right)$.

Pr o of. Let $y_{0}^{k}, \ldots, y_{n(k)}^{k}$ denote the vertices of the open simplex of $N(d)$ containing $q_{k}\left(x_{k}\right)$. By Definition 1 we have $d\left(x_{k}, y_{m}^{k}\right) \leq 1 / 3$, therefore the finite subset of $X$ formed by all the points $x_{k}$ and $y_{m}^{k}$ has diameter at most 1 and spans a simplex of $N(d)$.

We observe that for $d^{\prime} \geq d, N\left(d^{\prime}\right)$ is a subcomplex of $N(d)$, and for every barycentric map $q: X \rightarrow N\left(d^{\prime}\right)$ the composed map $X \rightarrow N\left(d^{\prime}\right) \hookrightarrow N(d)$ is also barycentric. We denote by $N_{X}$ the Vietoris nerve of the trivial metric $0 ; N_{X}$ contains all other Vietoris nerves.

LEMMA 2. For every metric $d$ on a space $X$ and every open covering $\mathcal{U}$ of $N(d)$ there is a metric $d^{\prime} \geq d$ on $X$ such that the inclusion map $N\left(d^{\prime}\right) \hookrightarrow N(d)$ is homotopic to a map $\gamma$ which maps each simplex of $N\left(d^{\prime}\right)$ to a $\mathcal{U}$-small subset of $N(d)$.

Proof. We may suppose that $\mathcal{U}$ consists of balls with respect to the canonical linear metric on $N(d)$. If $\left\{\psi_{\iota}\right\}$ is a locally finite partition of unity on $N(d)$ subordinate to a star refinement of $\mathcal{U}$, then $\varrho(x, y):=\sum_{\iota} \mid \psi_{\iota}(x)-$ $\psi_{\iota}(y) \mid$ is a metric on $N(d)$ such that every subset with diameter at most 1 is $\mathcal{U}$-small. We choose a barycentric map $q: X \rightarrow N(d)$ and set $d^{\prime}(x, y):=$ $\varrho(q(x), q(y))+3 d(x, y)$. If the points $x_{0}, \ldots, x_{n}$ span a simplex of $N\left(d^{\prime}\right)$ then in particular $\delta_{d}\left\{x_{0}, \ldots, x_{n}\right\} \leq 1 / 3$, and Lemma 1 tells that the points $x_{0}, \ldots, x_{n}, q\left(x_{0}\right), \ldots, q\left(x_{n}\right)$ belong to a single simplex of $N(d)$. Therefore we can define a homotopy $H: N\left(d^{\prime}\right) \times I \rightarrow N(d)$ by

$$
H(z, s):=s z+(1-s) \sum_{x \in X} \varphi_{x}(z) q(x) .
$$

Here $\varphi_{x}(z)$ means the barycentric coordinate of the point $z \in N\left(d^{\prime}\right)$ with respect to the vertex $x$. Then $H_{1}=\mathrm{id}$, and we claim that $\gamma:=H_{0}$ has the required property. Indeed, if the points $x_{0}, \ldots, x_{n}$ span a simplex $\sigma$ of $N\left(d^{\prime}\right)$, then $\varrho\left(q\left(x_{k}\right), q\left(x_{m}\right)\right) \leq 1$, and therefore the set $\left\{q\left(x_{0}\right), \ldots, q\left(x_{n}\right)\right\}$ is $\mathcal{U}$-small. Since $\gamma(\sigma)$ is the convex hull of this set it is also $\mathcal{U}$-small.

Since barycentric maps are not uniquely determined we cannot hope to build a morphism $X \rightarrow \mathbf{V}(X)$ in pro-Top out of them. In fact, such a morphism would give rise to a limit map $q: X \rightarrow \lim \mathbf{V}(X)=\bigcap_{d \in M_{X}} N(d)$ barycentric with respect to any metric $d$. If $X$ is completely regular, then the limit space is the set $X$ with the discrete topology, and $q$ would be the identity map, which is absurd if $X$ is not discrete. As remedy we perform the following construction: Let $\mathcal{M}_{X}$ denote the simplicial complex whose vertices are all metrics on $X$, a finite collection of metrics spanning a simplex if and only if it is linearly ordered by the natural order relation; by abuse of 
notation we denote the geometric realization of $\mathcal{M}_{X}$ by the same symbol. For any metric $d, \mathcal{M}(d)$ is the full subcomplex spanned by all metrics $d^{\prime} \geq d$. $\Lambda$ denotes the directed set of all finite subcomplexes of $\mathcal{M}_{X}$, ordered by inclusion, and for $K \in \Lambda$ we define $X_{K}$ to be the function space

$$
X_{K}:=\left\{\omega: K \rightarrow N_{X} \mid \forall d: \omega(K \cap \mathcal{M}(d)) \subseteq N(d)\right\}
$$

with the compact-open topology. By induction on the number of simplices of $K$ one can show that $X_{K}$ is an ANR-space. These spaces constitute an inverse system $\mathbf{V}^{\prime}(X):=\left\{f_{K}^{L}: X_{L} \rightarrow X_{K} \mid K \subseteq L \in \Lambda\right\}$, whose bonding maps are the appropriate restriction maps. $\mathbf{V}^{\prime}(X)$ depends functorially on $X$.

Proposition 2. In the Steenrod homotopy category $\mathbf{V}^{\prime}(X)$ is naturally isomorphic to the Vietoris system $\mathbf{V}(X)$.

Proof. The subset $\Lambda_{0} \subseteq \Lambda$ formed by all finite, full subcomplexes $K \subseteq \mathcal{M}_{X}$ which contain a largest vertex $d_{K}$ is cofinal, and so is the image of the nondecreasing index map $\Lambda_{0} \rightarrow M_{X}, K \mapsto d_{K}$. For each $K \in \Lambda_{0}$ let $i_{K}$ : $N\left(d_{K}\right) \hookrightarrow X_{K}$ be the natural embedding onto the set of constant mappings. The system of these maps determines a level morphism $\mathbf{i}: \mathbf{V}(X) \rightarrow \mathbf{V}^{\prime}(X)$ in pro-Top, and since each $i_{K}$ is a strong deformation retraction, $\mathbf{i}$ is a trivial cofibration.

For every $d \in M_{X}$ we choose a fixed barycentric map $q_{d}: X \rightarrow N(d)$, and for every simplex $\sigma$ of $\mathcal{M}_{X}$, given as a sequence of metrics $d_{0} \leq \ldots \leq d_{n}$ with corresponding barycentric maps $q_{k}: X \rightarrow N\left(d_{k}\right) \subseteq N\left(d_{0}\right)$, we denote by $\Gamma_{\sigma}: X \times \Delta^{n} \rightarrow N\left(d_{0}\right)$ the map described in Proposition 1 . There is a family of maps $q_{K}: X \rightarrow X_{K}$ such that for $K=\sigma$ the map $q_{K}$ is the adjoint of $\Gamma_{\sigma}$ and such that for $K \subseteq L$ the condition $f_{K}^{L} q_{L}=q_{K}$ holds. This defines a morphism $\mathbf{q}: X \rightarrow \mathbf{V}^{\prime}(X)$ in pro-Top, which is natural up to homotopy.

Proposition 3. $\mathbf{q}: X \rightarrow \mathbf{V}^{\prime}(X)$ is a strong expansion.

From Propositions 2 and 3 we derive the main result:

THEOREM 1. For every space $X$ the Vietoris system $\mathbf{V}(X)$ is naturally isomorphic in the Steenrod homotopy category to a strong ANR-expansion of $X$.

Proof of Proposition 3. 1) Suppose a map $h: X \rightarrow P$ into an ANR-space $P$ is given; we construct a metric $d$ on $X$ and a map $g: N(d) \rightarrow$ $P$ with $g q_{d} \simeq h . P$ may be considered as a bounded subset of a normed vector space $Y$ such that $P$ is closed in its convex hull $Y_{0}$. There is a covering $\mathcal{U}$ of $P$ consisting of open, convex subsets of $Y_{0}$ which are small enough to ensure the existence of a retraction map $r: \cup \mathcal{U} \rightarrow P$. As in the proof of Proposition 2 we construct a metric $\varrho$ on $P$ such that every subset of $P$ 
with diameter not exceeding 1 is $\mathcal{U}$-small. We set $d(x, y):=\varrho(h(x), h(y))$ and define $g^{\prime}: N(d) \rightarrow \bigcup \mathcal{U}$ by

$$
g^{\prime}(z):=\sum_{x \in X} \varphi_{x}(z) h(x),
$$

$\varphi_{x}$ being the barycentric coordinate function with respect to the vertex $x$. Then $g^{\prime}$ is continuous because $h(X)$ is bounded. We set $g:=r g^{\prime}: N(d) \rightarrow P$. Let us consider a point $x \in X$ and denote by $A_{x}$ the finite set of points $y \in X$ with $\varphi_{y}\left(q_{d}(x)\right)>0$. Lemma 1 ensures that $\{x\} \cup A_{x}$ spans a simplex of $N(d)$, hence $\{h(x)\} \cup h\left(A_{x}\right)$ is $\mathcal{U}$-small and therefore the points $h(x)$ and $g^{\prime}\left(q_{d}(x)\right)$ are $\mathcal{U}$-near. Now we can define a homotopy $H: h \simeq g q_{d}$ by $H(x, s):=r\left(s g^{\prime}\left(q_{d}\right)+(1-s) h(x)\right)$.

2) We consider two maps $g_{0}, g_{1}: N(d) \rightarrow P$ into an ANR-space $P$ and a homotopy $H: g_{0} q_{d} \simeq g_{1} q_{d}$, these data being prescribed, and a metric $e \geq d$ at our disposal. Let $\chi_{d}^{e}: N(e) \hookrightarrow N(d)$ denote the natural inclusion map and let $\sigma \subseteq K$ denote the 1-simplex connecting $d$ and $e$. The map $\Gamma_{\sigma}: X \times \Delta^{1} \rightarrow N(d)$ may be considered as a homotopy $\Gamma_{\sigma}: q_{d} \simeq \chi_{d}^{e} q_{e}$. Now we claim that $e$ can be chosen such that there is a homotopy $G: g_{0} \chi_{d}^{e} \simeq g_{1} \chi_{d}^{e}$ with $g_{0} \Gamma_{\sigma} \circ G\left(q_{e} \times\right.$ id $) \circ g_{1} \Gamma_{\sigma}^{-1} \simeq H$.

To this end we take an embedding $P \subseteq Y$, a covering $\mathcal{U}$, a retraction map $r: \cup \mathcal{U} \rightarrow P$ and a metric $\varrho$ on $P$ as above; and in addition, taking into account Lemma 2 we assume that $g_{0}$ and $g_{1}$ map every simplex of $N(d)$ into a $\mathcal{U}$-small subset of $P$. Now we define a metric $e$ on $X$ by

$$
e(x, y):=\sup _{t \in I} \varrho(H(x, t), H(y, t))+3 d(x, y) .
$$

For abbreviation we set $q:=q_{d}, q^{\prime}:=q_{e}$ and define

$$
G(z, s):=\left\{\begin{array}{lr}
\varrho\left((1-3 s) g_{0}(z)+3 s \sum_{x \in X} \varphi_{x}(z) g_{0}(q(x))\right), \quad s \leq 1 / 3, \\
\varrho\left(\sum_{x \in X} \varphi_{x}(z) H(x, 3 s-1)\right), \quad 1 / 3 \leq s \leq 2 / 3, \\
\varrho\left((3 s-2) g_{1}(z)+3(1-s) \sum_{x \in X} \varphi_{x}(z) g_{1}(q(x))\right), \quad s \geq 2 / 3 .
\end{array}\right.
$$

We observe that if $x_{0}, \ldots, x_{n}$ are the vertices of the open simplex carrying $z$, then $\delta_{d}\left\{x_{0}, \ldots, x_{n}\right\} \leq 1 / 3$. Lemma 1 ensures the existence of a simplex of $N(d)$ containing $z$ and $q\left(x_{0}\right), \ldots, q\left(x_{n}\right)$, and therefore $g_{0}(z), g_{0}\left(q\left(x_{0}\right)\right), \ldots$ $\ldots, g_{0}\left(q\left(x_{n}\right)\right)$ are $\mathcal{U}$-near points of $P$. This shows that $G(z, s)$ is well defined for $s \leq 1 / 3$, and the other cases are handled similarly.

We claim that $G$ satisfies $G\left(q^{\prime} \times\right.$ id $) \simeq g_{0} \Gamma_{\sigma}^{-1} \circ H \circ g_{1} \Gamma_{\sigma}$. The left hand homotopy is given by

$$
\left\{\begin{array}{lr}
\varrho\left((1-3 s) g_{0}\left(q^{\prime}(y)\right)+3 s \sum_{x \in X} \varphi_{x}\left(q^{\prime}(y)\right) g_{0}(q(x))\right), & s \leq 1 / 3, \\
\varrho\left(\sum_{x \in X} \varphi_{x}\left(q^{\prime}(y)\right) H(x, 3 s-1)\right), & 1 / 3 \leq s \leq 2 / 3, \\
\varrho\left((3 s-2) g_{1}\left(q^{\prime}(y)\right)+3(1-s) \sum_{x \in X} \varphi_{x}\left(q^{\prime}(y)\right) g_{1}(q(x))\right), & s \geq 2 / 3,
\end{array}\right.
$$


and the right hand one by

$$
\begin{cases}g_{0}\left((1-3 s) q^{\prime}(y)+3 s q(y)\right), & s \leq 1 / 3, \\ H(y, 3 s-1), & 1 / 3 \leq s \leq 2 / 3, \\ g_{1}\left((3 s-2) q^{\prime}(y)+3(1-s) q(y)\right), & s \geq 2 / 3 .\end{cases}
$$

Now let $x_{0}, \ldots, x_{n}$ be the vertices of the open simplex of $N(e)$ carrying $q^{\prime}(y)$. Since $q^{\prime}$ is barycentric we have $\delta_{e}\left\{y, x_{0}, \ldots, x_{n}\right\} \leq 2 / 3$ and hence $\delta_{d}\left\{y, x_{0}, \ldots, x_{n}\right\} \leq 2 / 9$, and Lemma 1 implies that the points $q(y), q^{\prime}(y)$, $q\left(x_{0}\right), \ldots, q\left(x_{n}\right)$ belong to a common simplex of $N(d)$. This means that for $s \leq 1 / 3$ the points $g_{0}\left((1-3 s) q^{\prime}(y)+3 s q(y)\right)$ and $(1-3 s) g_{0}\left(q^{\prime}(y)\right)+$ $3 s \sum_{x \in X} \varphi_{x}\left(q^{\prime}(y)\right) g_{0}(q(x))$ are $\mathcal{U}$-near and hence can be joined by a linear path $\Phi(y, s,-)$ in $\bigcup \mathcal{U}$, and similarly for $1 / 3 \leq s \leq 2 / 3$ and $s \geq 2 / 3$. Then $r \Phi: X \times I^{2} \rightarrow P$ is a 2-homotopy connecting $G\left(q^{\prime} \times \mathrm{id}\right)$ and $g_{0} \Gamma_{\sigma}^{-1} \circ H \circ g_{1} \Gamma$.

3) Suppose we are given $K \in \Lambda$, two maps $g_{0}, g_{1}: X_{K} \rightarrow P \in \mathrm{ANR}$ and a homotopy $H: g_{0} q_{K} \simeq g_{1} q_{K}$; we have to construct $L \in \Lambda$ with $L \supseteq K$ and a homotopy $G: g_{0} f_{K}^{L} \simeq g_{1} f_{K}^{L}$ with $G\left(q_{L} \times\right.$ id $) \simeq H$. We may assume that the complex $K$ is full and contains a largest vertex $d$. Then the map $f_{d}^{K}: X_{K} \rightarrow X_{d}$ is a homotopy equivalence whose inverse $a: X_{d} \rightarrow X_{K}$ embeds $X_{d}$ in $X_{K}$ as a set of constant mappings and the homotopy $A$ : id $\simeq$ $a f_{d}^{K}$ is determined by a parameter transformation; the equation $f_{d}^{K} a=\mathrm{id}$ holds. Now we apply step 2 to the mappings $g_{0} a, g_{1} a: X_{d}=N(d) \rightarrow P$ and the homotopy $\left(g_{0} A\left(q_{K} \times \mathrm{id}\right)\right)^{-1} \circ H \circ\left(g_{1} A\left(q_{K} \times \mathrm{id}\right)\right): g_{0} a q_{d} \simeq g_{1} a q_{d}$ and obtain a metric $e \geq d$ and a homotopy $G^{\prime}: g_{0} a \chi_{d}^{e} \simeq g_{1} a \chi_{d}^{e}$ with $\left(g_{0} A\left(q_{K} \times \mathrm{id}\right)\right)^{-1} \circ H \circ\left(g_{1} A\left(q_{K} \times \mathrm{id}\right)\right) \simeq g_{0} a \Gamma_{\sigma} \circ G^{\prime}\left(q_{e} \times \mathrm{id}\right) \circ g_{1} a \Gamma_{\sigma}^{-1}$. Let $L$ be the full subcomplex generated by $K$ and the vertex $e$. We define a homotopy $F: f_{d}^{L} \simeq \chi_{d}^{e} f_{e}^{L}$ by $F(\omega, s):=\omega(s e+(1-s) d)$ and observe that $F\left(q_{L} \times \mathrm{id}\right)=\Gamma_{\sigma}$. Now the homotopy $G:=g_{0}\left(A\left(f_{K}^{L} \times \mathrm{id}\right) \circ a F\right) \circ G^{\prime}\left(f_{e}^{L} \times\right.$ id) $\circ g_{1}\left(A\left(f_{K}^{L} \times \mathrm{id}\right) \circ a F\right)^{-1}: g_{0} f_{K}^{L} \simeq g_{1} f_{K}^{L}$ has the required property.

2. Strong homology. Let $\mathbf{C}_{*}$ be a filtered chain complex of $R$-modules, which means we are given a chain complex $C_{*}$ over a commutative, unitarian ring $R$ with a decreasing filtration of subcomplexes $C_{*}(\lambda)$, where $\lambda$ ranges over some nonempty directed set $\Lambda$. This ordered set determines a simplicial complex whose simplices are the finite increasing sequences of indices; we denote by $\Lambda_{*}$ the corresponding chain complex based on ordered simplices with coefficients in $R . \Lambda_{*}$ is filtered by the subcomplexes $\Lambda_{*}(\lambda)$ of chains of simplices $\left\langle\mu_{0} \leq \ldots \leq \mu_{p}\right\rangle$ with $\mu_{0} \geq \lambda$.

Definition 2. The total complex $\Gamma_{*}$ of $\mathbf{C}_{*}$ has as $n$-simplices the graded homomorphisms of degree $n, \varphi \in \operatorname{Hom}\left(\Lambda_{*}, C_{*+n}\right)$, with $\varphi\left(\Lambda_{*}(\lambda)\right) \subseteq C_{*+n}(\lambda)$ for each index $\lambda$. The differential of $\Gamma_{*}$ is defined by $(\partial \varphi)(x):=\partial(\varphi(x))-$ $(-1)^{\operatorname{deg} \varphi} \varphi(\partial x)$. 
In plain terms an $n$-chain of $\Gamma_{*}$ is a function assigning to each sequence of indices $\lambda_{0} \leq \ldots \leq \lambda_{p}$ a $p+n$-chain of $C_{*}\left(\lambda_{0}\right)$, so Definition 2 coincides with the one given in [17, Section 3] for arbitrary inverse systems of chain complexes. We restrict our attention to filtered complexes, because it simplifies notation and is sufficient for the applications we have in mind. The differential defined in [17] coincides with ours apart from a change of sign, which does not affect the homology. Our sign convention is taken from the function complex definition familiar in homological algebra. Interpreting total complexes as function complexes will turn out to be advantageous when it comes to induced mappings.

Lemma 3. For every directed set $\Lambda$ the chain complex $\Lambda_{*}$ has the homology of a point.

Proof. We consider an $n$-chain $x=\sum_{i} r_{i} \sigma_{i}$ of $\Lambda$; for $n=0$ we suppose that $x$ is reduced, $\sum_{i} r_{i}=0$, and for $n>0$ we assume $\partial x=0$. If $\lambda$ is an element of $\Lambda$ dominating all vertices of the simplices $\sigma_{i}$, then we can form $n+1$-simplices $\widehat{\sigma}_{i}$ by adding the vertex $\lambda$ to $\sigma_{i}$ and set $y:=(-1)^{n+1} \sum_{i} r_{i} \widehat{\sigma}_{i}$. This implies $\partial y=x$.

Suppose we are given two filtered complexes $\mathbf{C}_{*}^{\prime}$ and $\mathbf{C}_{*}$ and a chain map $f: C_{*}^{\prime} \rightarrow C_{*}$ such that for each $\lambda \in \Lambda$ there exists $\alpha(\lambda) \in \Lambda^{\prime}$ with

$$
f\left(C_{*}^{\prime}(\alpha(\lambda))\right) \subseteq C_{*}(\lambda) .
$$

The map $\alpha$ is not assumed to be monotone. Lemma 3 tells us that for each $\lambda \in \Lambda$ the chain complex $\sum_{\mu>\lambda} \Lambda_{*}^{\prime}(\alpha(\mu))$ has the homology of a point, because it is the complex of the directed set $\left\{\lambda^{\prime} \in \Lambda \mid \exists \mu \geq \lambda: \lambda^{\prime} \geq \alpha(\mu)\right\}$. Hence we can construct a chain map $\beta: \Lambda_{*} \rightarrow \Lambda_{*}^{\prime}$ with

$$
\beta\left(\Lambda_{*}(\lambda)\right) \subseteq \sum_{\mu \geq \lambda} \Lambda_{*}^{\prime}(\alpha(\mu))
$$

for each $\lambda$, and any two of them are chain homotopic. Then we can define a chain map $F: \Gamma_{*}^{\prime} \rightarrow \Gamma_{*}$ by

$$
F(\varphi):=f \varphi \beta
$$

LEMMA 4. The chain homotopy class of $F$ does not depend on the particular choice of the maps $\alpha$ and $\beta$, as long as conditions (1) and (2) are satisfied. The assignment $f \mapsto F$ is functorial up to chain homotopy.

This follows easily from Lemma 3.

THEOREM 2. If for any two indices $\lambda_{0} \in \Lambda$ and $\lambda_{0}^{\prime} \in \Lambda^{\prime}$ there are $\lambda_{1} \geq \lambda_{0}$ and $\lambda_{1}^{\prime} \geq \lambda_{0}^{\prime}$ with $f\left(C_{*}^{\prime}\left(\lambda_{1}^{\prime}\right)\right) \subseteq C_{*}\left(\lambda_{1}\right)$ such that $f: C_{*}^{\prime}\left(\lambda_{1}^{\prime}\right) \rightarrow C_{*}\left(\lambda_{1}\right)$ induces isomorphisms of all homology groups, then $F: \Gamma_{*}^{\prime} \rightarrow \Gamma_{*}$ shares this property. 
Pro o f. In the special case of $\Lambda=\Lambda^{\prime}$, for all $\lambda \in \Lambda$ we have $f\left(C_{*}^{\prime}(\lambda)\right) \subseteq$ $C_{*}(\lambda)$ and $f: C_{*}^{\prime}(\lambda) \rightarrow C_{*}(\lambda)$ induces isomorphisms of all homology groups. Using a mapping cone argument (see Spanier [26, Lemma 4.6.9]) the proof that $F$ induces an isomorphism of homology reduces to showing that $\Gamma_{*}$ is acyclic whenever each $C_{*}(\lambda)$ is acyclic. But this follows readily from the definition of the differential.

To deal with the general case we consider the set $M \subseteq \Lambda^{\prime} \times \Lambda$ of all pairs $\left(\lambda^{\prime}, \lambda\right)$ with $f\left(C_{*}^{\prime}\left(\lambda^{\prime}\right)\right) \subseteq C_{*}(\lambda)$ such that $f: C_{*}^{\prime}\left(\lambda^{\prime}\right) \rightarrow C_{*}(\lambda)$ induces an isomorphism of homology, and let $\beta: M \rightarrow \Lambda^{\prime}, \gamma: M \rightarrow \Lambda$ denote the projection maps. The assumption ensures that the set $M$ is directed by the product order, that $\beta(M)$ is cofinal in $\Lambda^{\prime}$ and that $\gamma(M)$ is cofinal in $\Lambda$. We introduce new filtrations on our two chain complexes indexed with $M$ by setting $\bar{C}_{*}^{\prime}\left(\lambda^{\prime}, \lambda\right):=C_{*}^{\prime}\left(\lambda^{\prime}\right)$ and $\bar{C}_{*}\left(\lambda^{\prime}, \lambda\right):=C_{*}(\lambda)$. From the corresponding total complexes we can build the following, chain homotopy commutative diagram:

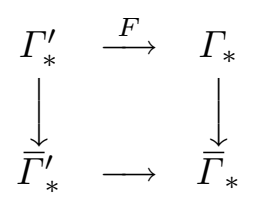

The lower horizontal arrow is of the special form treated before, and the vertical arrows are chain homotopy equivalences by Lemma 4 , and consequently $F$ induces isomorphisms of all homology groups.

Supplied with these tools we can now start to define the strong homology groups of a topological pair $(X, A)$ with $X \neq \emptyset$. In the set $M_{X}$ of all metrics on $X$ we consider the subset $M_{(X, A)}$ of all metrics with trivial trace on $A$. We denote by $\widetilde{\mathbf{C}}_{*}(X, A ; G)$ the reduced oriented simplicial chain complex of the complex $N_{X}$ with coefficients in an $R$-module $G$, filtered by the subcomplexes determined by $N(d)$ for $d \in M_{(X, A)}$. We emphasize that $\widetilde{\mathbf{C}}_{*}(X, A ; G)$ is based upon oriented simplices of the form $\left\langle x_{0}, \ldots, x_{n}\right\rangle$ with $x_{i} \in X$ (see [26, p. 158]), and that we do not use the more general singular simplices of the geometric realization of $N_{X}$ (cf. [26, p. 173]). Thus an $n$-chain of $\widetilde{C}_{*}(X, A ; G)$ is a finite linear combination of the form

$$
\sum_{i} g_{i}\left\langle x_{0}^{i}, \ldots, x_{n}^{i}\right\rangle
$$

with $g_{i} \in G$ and $x_{j}^{i} \in X$, for $n=0$ we have $\sum_{i} g_{i}=0$, and the chain belongs to $\widetilde{C}_{*}(d)$ if the $d$-diameter of the sets $\left\{x_{0}^{i}, \ldots, x_{n}^{i}\right\}$ does not exceed 1 .

Definition 3 . The reduced strong homology $H_{*}^{\mathrm{s}}(X, A ; G)$ of a topological pair $(X, A), X \neq \emptyset$, with coefficients in an $R$-module $G$ is the homology 
of the total complex of $\widetilde{\mathbf{C}}_{*}(X, A ; G)$. Induced mappings are defined as in Section 1.

Remark. a) Strong homology for arbitrary spaces has been introduced independently by Miminoshvili in [22] and by Lisica and Mardešić in [11, 12, 13] with further investigations in $[19,17,16]$. In [12] it is proved that strong homology of inverse systems of spaces is a functor on the coherent prohomotopy category, and hence, by [8], on the Steenrod homotopy category. The strong homology of a space $X$ is defined as the strong homology of a resolution $\widehat{\mathbf{X}}$ of $X$. By [14], $\widehat{\mathbf{X}}$ is a strong expansion of $X$, and since any two strong expansions are isomorphic in the Steenrod homotopy category it is isomorphic to the Vietoris system $\mathbf{V}(X)$. The strong homology of this inverse system is our $H_{*}^{\mathrm{s}}(X ; G)$ with oriented simplices replaced by singular simplices, but Theorem 2 permits this replacement without affecting the homology.

b) For compact Hausdorff spaces $X$ our $H_{*}^{\mathrm{s}}(X ; G)$ is isomorphic to Massey's homology [20]. The proof will be given in Section 3.

The Eilenberg-Steenrod axioms are ready at our hand. The proof of the dimension axiom is left to the reader, but he should observe that we do not claim $H_{n}^{\mathrm{s}}=0$ for $n<0$ (for the reason we refer to [17, Theorem 3]), and that our strong homology functor is reduced.

\section{THEOREM 3.}

$$
H_{n}^{\mathrm{s}}\left(S^{0} ; G\right)= \begin{cases}G, & n=0, \\ 0, & n \neq 0 .\end{cases}
$$

Here comes the excision axiom in the form proved by Mardešić and Miminoshvili in [16, Theorem 4]:

Theorem 4. For any topological pair $(X, A)$ with $A \neq \emptyset$ the quotient map $p:(X, A) \rightarrow(X / A, *)$ induces isomorphisms of the strong homology groups $H_{*}^{\mathrm{s}}(p): H_{*}^{\mathrm{s}}(X, A ; G) \rightarrow H_{*}^{\mathrm{s}}(X / A, * ; G)$.

In the proof we will make use of the following observation:

Lemma 5. Consider two contiguous simplicial mappings $f, g: K \rightarrow L$ between simplicial complexes (see [26, p. 130]) and a subcomplex $K^{\prime} \subseteq K$ on which $f$ and $g$ coincide. Then the induced chain maps $\widetilde{C}_{*}(f), \widetilde{C}_{*}(g)$ : $\widetilde{C}_{*}(K ; G) \rightarrow \widetilde{C}_{*}(L ; G)$ can be connected by a chain homotopy @ which vanishes on $\widetilde{C}_{*}\left(K^{\prime} ; G\right)$.

Pro of. We introduce a linear order on the vertex set of $K$ and define

$$
\varrho\left(k_{0}<k_{1}<\ldots<k_{n}\right):=\sum_{i=0}^{n}(-1)^{i}\left\langle f\left(k_{0}\right), \ldots, f\left(k_{i}\right), g\left(k_{i}\right), \ldots, g\left(k_{n}\right)\right\rangle .
$$


For $k_{i} \in K^{\prime}$ the simplex on the right hand side is degenerate and therefore determines the zero element of $\widetilde{C}_{*}(L ; G)$.

Now the proof of Theorem 4 is a straightforward application of Theorem 2. In fact, the chain map $\widetilde{\mathbf{C}}_{*}(p): \widetilde{\mathbf{C}}_{*}(X, A ; G) \rightarrow \widetilde{\mathbf{C}}_{*}(X / A, * ; G)$ is of the special form mentioned at the beginning of the proof of Theorem 2 , because $p^{*}: M_{(X / A, *)} \rightarrow M_{(X, A)}$ is one-to-one. Furthermore, the vertex map $p_{*}: N\left(p^{*} d\right) \rightarrow N(d)$ is surjective, and any sequence of points $x_{0}, \ldots, x_{n} \in X$ determines a simplex of $N\left(p^{*} d\right)$ if and only if $p\left(x_{0}\right), \ldots, p\left(x_{n}\right)$ determines a simplex of $N(d)$. Hence $p_{*}: N\left(p^{*} d\right) \rightarrow N(d)$ is invertible up to contiguity, and by Lemma $5, \widetilde{C}_{*}(p): \widetilde{C}_{*}\left(p^{*} d\right) \rightarrow \widetilde{C}_{*}(d)$ is a chain homotopy equivalence.

Our next topic is the exact homology sequence of a topological pair $(X, A)$ with normally embedded subspace $A \neq \emptyset$. We recall that a subspace $A$ of a topological space $X$ is normally embedded if every metric on $A$ can be extended over $X$; the equivalence of this characterization with the familiar covering property [18, p. 89] is proved in [1, Theorem 14.7]. Clearly for any increasing sequence of metrics on $A$ we can inductively construct an increasing sequence of extensions over $X$, so that the restriction map induces a surjection of the complex of the directed set $M_{X}$ onto that of $M_{A}$. Therefore the inclusion map $i: A \hookrightarrow X$ induces a monomorphism $\Gamma_{*}(i): \Gamma_{*}(A) \rightarrow \Gamma_{*}(X)$ of the total complexes, by means of which we consider $\Gamma_{*}(A)$ as a subcomplex of $\Gamma_{*}(X)$. Now we remind ourselves of the quotient map $p:(X, A) \rightarrow(X / A, *)$; we have already learned that $\Gamma_{*}(p): \Gamma_{*}(X, A) \rightarrow \Gamma_{*}(X / A)$ induces an isomorphism of homology. $\Gamma_{*}(p): \Gamma_{*}(X) \rightarrow \Gamma_{*}(X / A)$ maps $\Gamma_{*}(A)$ to 0 and hence determines a map $q: \Gamma_{*}(X) / \Gamma_{*}(A) \rightarrow \Gamma_{*}(X / A)$.

THEOREM 5. For every topological pair $(X, A)$ with nonempty, normally embedded subspace the quotient chain map $q: \Gamma_{*}(X) / \Gamma_{*}(A) \rightarrow \Gamma_{*}(X / A)$ induces isomorphisms of the homology groups; therefore the short exact sequence of chain complexes

$$
0 \rightarrow \Gamma_{*}(A) \rightarrow \Gamma_{*}(X) \rightarrow \Gamma_{*}(X) / \Gamma_{*}(A) \rightarrow 0
$$

provides us with a natural exact homology sequence

$$
\ldots \rightarrow H_{n}^{\mathrm{s}}(A ; G) \rightarrow H_{n}^{\mathrm{s}}(X ; G) \rightarrow H_{n}^{\mathrm{s}}(X, A ; G) \rightarrow H_{n-1}^{\mathrm{s}}(A ; G) \rightarrow \ldots
$$

For the proof we need the following variant of Morita's lemma (cf. [18, p. 112]):

LEMMA 6. Suppose we are given a filtered chain complex $\mathbf{C}_{*}$ and a subcomplex $C_{*}^{\prime} \subseteq C_{*}$; on $C_{*}^{\prime}$ we consider the filtration $C_{*}^{\prime}(\lambda)=C_{*}^{\prime} \cap C_{*}(\lambda)$. If for every index $\lambda$ there are $\mu \geq \lambda$, a chain map $\tau: C_{*}(\mu) \rightarrow C_{*}^{\prime}(\lambda)$ extending the inclusion map $C_{*}^{\prime}(\mu) \hookrightarrow C_{*}^{\prime}(\lambda)$ and a chain homotopy $\varrho$ from $\tau$ to the 
inclusion homomorphism $C_{*}(\mu) \hookrightarrow C_{*}(\lambda)$, with $\varrho\left(C_{*}^{\prime}(\mu)\right) \subseteq C_{*}^{\prime}(\lambda)$, then the inclusion chain map of the total complexes $\Gamma_{*}^{\prime} \hookrightarrow \Gamma_{*}$ induces isomorphisms of the homology groups.

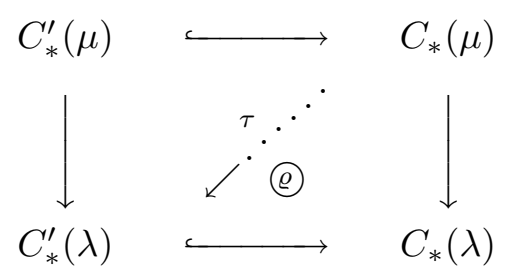

Proof. We consider the quotient complex $C_{*}^{\prime \prime}:=C_{*} / C_{*}^{\prime}$ filtered by the subcomplexes $C_{*}^{\prime \prime}(\lambda):=C_{*}(\lambda) / C_{*}^{\prime}(\lambda)$. If $\lambda$ and $\mu$ are as in the assumption, then the inclusion chain map $C_{*}^{\prime \prime}(\mu) \hookrightarrow C_{*}^{\prime \prime}(\lambda)$ is nullhomotopic. Now it suffices to show that the total complex $\Gamma_{*}^{\prime \prime}$ is acyclic. By Theorem 2 we may assume that the index set $\Lambda$ is cofinite, because else we could reindex $\mathbf{C}_{*}^{\prime \prime}$ with the directed set of all finite subsets of $\Lambda$ containing a greatest element. If $\Lambda$ is cofinite, then we can construct a nondecreasing index map $\alpha: \Lambda \rightarrow \Lambda$ such that $C_{*}^{\prime \prime}(\alpha(\lambda)) \hookrightarrow C_{*}^{\prime \prime}(\lambda)$ is nullhomotopic. Suppose $\varphi$ is a cycle of $\Gamma_{*}^{\prime \prime}$; by induction on $\operatorname{deg} x, x \in \Lambda_{*}$, we can construct elements $\psi(x) \in C_{*}^{\prime \prime}$ with

$$
\begin{gathered}
\partial(\psi(x))=\varphi\left(\alpha_{*} x\right)-(-1)^{\operatorname{deg} \varphi} \psi(\partial x), \\
\psi(x) \in C_{*}^{\prime \prime}(\alpha(\lambda)) \quad \text { for } x \in \Lambda_{*}(\lambda),
\end{gathered}
$$

because the right hand side of equation (4) is a cycle of $C_{*}^{\prime \prime}(\alpha(\lambda))$ and hence a boundary in $C_{*}^{\prime \prime}(\lambda) ; \alpha_{*}: \Lambda_{*} \rightarrow \Lambda_{*}$ is the chain map induced by our nondecreasing index map. Then $\partial \psi=\alpha^{*} \varphi$, but by Lemma $4, \alpha^{*} \varphi$ and $\varphi$ are homologous.

Lemma 7. Let $A \subseteq X$ be a nonempty, normally embedded subspace. Denote by $\mathbf{C}_{*}^{0}(X, A ; G) \subseteq \widetilde{\mathbf{C}}_{*}(X, A ; G)$ the filtered subcomplex such that for each metric $d \in M_{X}$ the subcomplex $C_{*}^{0}(d) \subseteq \widetilde{C}_{*}(d)$ is determined by the simplices $\left\langle x_{0}, \ldots, x_{n}\right\rangle$ with $d\left(x_{i}, A\right) \leq 2$ for all $i$, and denote by $\Gamma_{*}^{0}(X, A) \subseteq \Gamma_{*}(X, A)$ the derived total complex. Then the natural map $\Gamma_{*}(A) \rightarrow \Gamma_{*}^{0}(X, A)$ induces isomorphisms of all homology groups.

Proof. Since $A$ is normally embedded in $X$ we can reindex $\widetilde{\mathbf{C}}_{*}(A ; G)$ using $M_{X}$ as index set, and then we are in a position to apply Lemma 6 . Let $d \in M_{X}$ be given. For any $x \in X$ with $7 d(x, A) \leq 2$ we choose $\alpha(x) \in A$ with $d(x, \alpha(x)) \leq 3 / 7$, assuming $\alpha(x)=x$ for $x \in A$. Then $\alpha$ may be considered as simplicial map $\alpha: N^{0}(7 d) \rightarrow N\left(d_{\mid A}\right)$, where $N^{0}(7 d) \subseteq N(7 d)$ is the full subcomplex determined by all vertices $x$ with $7 d(x, A) \leq 2$, which operates as identity on $N\left(7 d_{\mid A}\right)$ and which is contiguous in $N^{0}(d)$ to the inclusion map $N^{0}(7 d) \hookrightarrow N^{0}(d)$. Now $\alpha$ induces our chain map $\tau: \widetilde{C}_{*}^{0}(7 d) \rightarrow \widetilde{C}_{*}\left(d_{\mid A}\right)$, and the existence of $\varrho$ follows from Lemma 5 . 
Proof of Theorem 5. We consider the following commutative diagram:

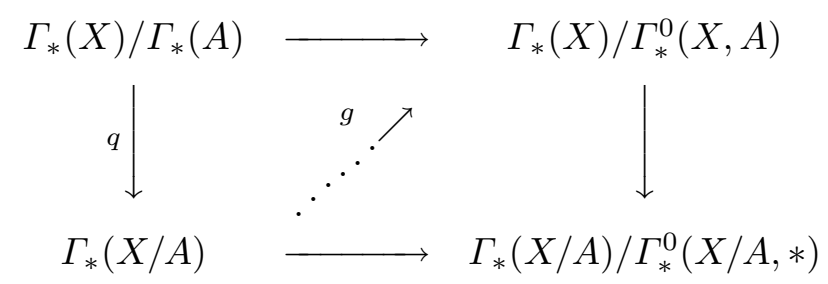

The vertical maps are induced by $p: X \rightarrow X / A$ and the horizontal maps are algebraic quotient maps. By Lemma 7 the horizontal maps induce an isomorphism of homology, because the one-point subspace $*$ is certainly normally embedded in $X / A$. If we can construct a filler $g$ to the diagram above, then $q$ induces an isomorphism of homology. We start by constructing a nondecreasing index map $M_{X} \rightarrow M_{X / A}, d \mapsto d^{\prime}$, with

$$
d^{\prime}(p(x), p(y)):=\min (d(x, y), d(x, A)+d(y, A)) .
$$

Furthermore, we assign to each metric $d \in M_{X}$ a graded homomorphism $\vartheta_{d}: \widetilde{C}_{*}(X / A ; G) \rightarrow \widetilde{C}_{*}(X ; G)$, which is defined on the generating simplices $\sigma=\left\langle p\left(x_{0}\right), \ldots, p\left(x_{n}\right)\right\rangle$ by $\vartheta_{d}(\sigma):=\left\langle x_{0}, \ldots, x_{n}\right\rangle$ if $d\left(x_{i}, A\right) \geq 1$ for all $i$, and $\vartheta_{d}(\sigma)=0$ else. For $d^{\prime}\left(p\left(x_{i}\right), p\left(x_{j}\right)\right) \leq 1$ and $d\left(x_{i}, A\right) \geq 1, d\left(x_{j}, A\right) \geq 1$ we necessarily have $d\left(x_{i}, x_{j}\right)=d^{\prime}\left(p\left(x_{i}\right), p\left(x_{j}\right)\right) \leq 1$ and hence

$$
\vartheta_{d}\left(\widetilde{C}_{*}\left(d^{\prime}\right)\right) \subseteq \widetilde{C}_{*}(d)
$$

$\vartheta_{d}$ is not a chain map, but at least we have

$$
\left(\partial \vartheta_{d}-\vartheta_{d} \partial\right)\left(\widetilde{C}_{*}\left(d^{\prime}\right)\right) \subseteq C_{*}^{0}(d),
$$

because the only possibility for $\partial \vartheta_{d}(\sigma)$ and $\vartheta_{d} \partial(\sigma)$ to be distinct from one another is the existence of some $i$ with $d\left(x_{i}, A\right)<1$, and then all $x_{j}$ necessarily satisfy $d\left(x_{j}, A\right) \leq 2$. Similarly we see that

$$
\begin{aligned}
\left(\vartheta_{d} p_{*}-\mathrm{id}\right)\left(\widetilde{C}_{*}(d)\right) \subseteq C_{*}^{0}(d), \\
\left(p_{*} \vartheta_{d}-\mathrm{id}\right)\left(\widetilde{C}_{*}\left(d^{\prime}\right)\right) \subseteq C_{*}^{0}\left(d^{\prime}\right), \\
\left(\vartheta_{d_{0}}-\vartheta_{d_{1}}\right)\left(\widetilde{C}_{*}\left(d_{1}^{\prime}\right)\right) \subseteq C_{*}^{0}\left(d_{0}\right) \text { for } d_{0} \leq d_{1} .
\end{aligned}
$$

Equations (8), (9) and (12) allow us to define a chain map $g: \Gamma_{*}(X / A) \rightarrow$ $\Gamma(X) / \Gamma_{*}^{0}(X, A)$ by

$$
g(\psi)\left\langle d_{0} \leq \ldots \leq d_{p}\right\rangle:=\vartheta_{d_{0}}\left(\psi\left\langle d_{0}^{\prime} \leq \ldots \leq d_{p}^{\prime}\right\rangle\right),
$$

and by equations (10) and (11), $g$ fits commutatively into diagram (6).

Now we prove at last that our homology functor $H_{*}^{\mathrm{s}}$ is homotopy invariant: 
TheOREM 6. For every topological pair $(X, A)$ the projection map $p$ : $(X \times I, A \times I) \rightarrow(X, A)$ induces isomorphisms of the homology groups $H_{*}^{\mathrm{s}}(p): H_{*}^{\mathrm{s}}(X \times I, A \times I ; G) \rightarrow H_{*}^{\mathrm{s}}(X, A ; G)$.

Pr o of. We want to apply Theorem 2 and therefore we need some special metrics on $X \times I$ (which will serve us as substitutes for stacked coverings). To any metric $d$ on $X$ and any map $\alpha: X \rightarrow \mathbb{R}_{+}$into the positive reals we assign a metric $d^{\alpha}$ on $X \times I$ by setting

$$
d^{\alpha}(x, s ; y, t):=\max (d(x, y),|\alpha(x) s-\alpha(y) t|) .
$$

If $d$ and $\alpha$ vanish on $A$, then $d^{\alpha}$ also has trivial trace on $A$. We claim:

(a) For every metric $d^{\prime} \in M_{(X \times I, A \times I)}$ there exist a metric $d \in M_{(X, A)}$ and a map $\alpha: X \rightarrow \mathbb{R}_{+}$with $\alpha_{\mid A}=0$ and $d^{\alpha}(x, s ; y, t) \leq 1 \Rightarrow d^{\prime}(x, s ; y, t) \leq 1$.

(b) $p_{*}: N\left(d^{\alpha}\right) \rightarrow N(d)$ induces an isomorphism of homology.

(a), (b) and Theorem 2 imply that $H_{*}^{\mathrm{s}}(p)$ is an isomorphism, because for $d_{0} \in M_{(X, A)}$ and $d_{0}^{\prime} \in M_{(X \times I, A \times I)}$ we can choose $d_{1} \in M_{(X, A)}, d_{1} \geq d_{0}$, and $\alpha: X \rightarrow \mathbb{R}_{+}$with property (a). Then we set $d_{1}^{\prime}:=\max \left(d_{0}^{\prime}, d_{1}^{\alpha}\right)$ to get a metric $d_{1}^{\prime} \geq d_{0}^{\prime}$ whose Vietoris nerve coincides with that of $d_{1}^{\alpha}$, so that by (b), $p_{*}: N\left(d_{1}^{\prime}\right) \rightarrow N\left(d_{1}\right)$ induces isomorphisms of the homology groups.

To prove (a) we set $d_{0}(x, y):=\sup _{t} d^{\prime}(x, t ; y, t)$ and consider the following subsets of $X$ :

$$
\begin{aligned}
& U_{n}:= \begin{cases}\left\{x \in X \mid d_{0}(x, A) \leq 1 / 4\right\}, & n=0 \\
\left\{x \in X \backslash A|| s-t \mid \leq 2 / n \Rightarrow d^{\prime}(x, s ; x, t) \leq 1 / 2\right\}, & n \geq 1\end{cases} \\
& V_{n}:= \begin{cases}\left\{x \in X \mid d_{0}(x, A) \leq 1 / 8\right\}, & n=0 \\
\left\{x \in X \backslash A|| s-t \mid \leq 2 / n \Rightarrow d^{\prime}(x, s ; x, t) \leq 1 / 4\right\}, & n \geq 1\end{cases}
\end{aligned}
$$

By Lebesgue's Theorem the sets $V_{n}$ cover $X$, and each $V_{n}$ is contained in the interior of $U_{n}$ with respect to the metric $d_{0}$. Therefore there exists a continuous, locally finite partition of unity $\left\{\varphi_{n}\right\}$ on the pseudometric space $\left(X, d_{0}\right)$ with $\left.\left.\varphi_{n}^{-1}\right] 0,1\right] \subseteq U_{n}$. All requirements of (a) are satisfied if we define

$$
\alpha(x):=\sum_{n=0}^{\infty} n \varphi_{n}(x), \quad d(x, y):=\max \left(2 d_{0}(x, y),|\alpha(x)-\alpha(y)|\right) .
$$

To prove (b) we consider the map $\chi: \widetilde{C}_{*}(N(d) ; G) \rightarrow \widetilde{C}_{*}\left(N\left(d^{\alpha}\right) ; G\right)$ induced by the vertex map $x \mapsto(x, 0)$. Clearly $\chi$ is right inverse to $p$ : $\widetilde{C}_{*}\left(N\left(d^{\alpha}\right) ; G\right) \rightarrow \widetilde{C}_{*}(N(d) ; G)$, and we are going to construct a chain homotopy $\varrho$ connecting $\chi p$ with the identity map. We define functions $f_{x}^{n}: I \rightarrow I$ for $x \in X$ and $0 \leq n<\infty$ by

$$
f_{x}^{n}(t):= \begin{cases}\alpha(x)^{-1} n, & n<\alpha(x) t \\ t, & n \geq \alpha(x) t, n>0 \\ 0, & n=0\end{cases}
$$


and observe that $|\alpha(x) s-\alpha(y) t| \leq 1$ implies $\left|\alpha(x) f_{x}^{n}(s)-\alpha(y) f_{y}^{n}(t)\right| \leq 1$ and $\left|\alpha(x) f_{x}^{n}(s)-\alpha(y) f_{y}^{n+1}(t)\right| \leq 1$; furthermore, $f_{x}^{0}(t)=0$ and $f_{x}^{n}(t)=t$ for $n>\alpha(x)$. Let $F_{n}: N\left(d^{\alpha}\right) \rightarrow N\left(d^{\alpha}\right)$ be the simplicial map $F_{n}(x, t):=$ $\left(x, f_{x}^{n}(t)\right)$; then $F_{n}$ and $F_{n+1}$ are contiguous and coincide for $n>\alpha(x)$. By Lemma 5 the induced chain mappings $\widetilde{C}_{*}\left(F_{n}\right), \widetilde{C}_{*}\left(F_{n+1}\right): \widetilde{C}_{*}\left(d^{\alpha}\right) \rightarrow$ $\widetilde{C}_{*}\left(d^{\alpha}\right)$ can be connected by a chain homotopy $\varrho_{n}, \partial \varrho_{n}+\varrho_{n} \partial=\widetilde{C}_{*}\left(F_{n+1}\right)-$ $\widetilde{C}_{*}\left(F_{n}\right)$, with $\varrho_{n}\left\langle\left(x_{0}, t_{0}\right), \ldots,\left(x_{m}, t_{m}\right)\right\rangle=0$ for $n>\max _{i} \alpha\left(x_{i}\right)$. Therefore we can define $\varrho:=\sum_{n=0}^{\infty} \varrho_{n}$, because the apparently infinite sum terminates for every chain of the domain. Then $\varrho$ is a chain homotopy: $\partial \varrho+\varrho \partial=$ id $-\chi p$.

3. Strong homology with compact supports. There is a natural notion of support of a chain $\varphi \in \Gamma_{*}(X, A ; G)$ : If for each increasing sequence $\mu=\left\langle d_{0} \leq \ldots \leq d_{p}\right\rangle$ of metrics in $M_{(X, A)}$ we have $\varphi(\mu)=$ $\sum_{i} g_{i}^{\mu}\left\langle x_{\mu 0}^{i}, \ldots, x_{\mu, n+p}^{i}\right\rangle$, then $\operatorname{Supp}(\varphi)$ is the smallest closed subset of $X$ containing all the points $x_{\mu k}^{i}$ with $g_{i}^{\mu} \neq 0$. The chains with compact support form a subcomplex $\Gamma_{*}^{\mathrm{c}}(X, A ; G) \subseteq \Gamma_{*}(X, A ; G)$, whose homology is denoted by $H_{*}^{\mathrm{c}}(X, A ; G)$ and is called strong homology with compact supports. We are going to show that under some restrictions on $(X, A)$ its homology group can be obtained by taking direct limits.

Lemma 8. Every compact subspace A of a completely regular Hausdorff space $X$ is normally embedded.

Pro of. Let $d$ be a metric on $A, \widetilde{A}$ the metric space obtained by identifying points of distance 0 and $q: A \rightarrow \widetilde{A}$ the quotient map. Since $\widetilde{A}$ is compact metric there is an embedding $\varphi: \widetilde{A} \hookrightarrow Q$ into the Hilbert cube. $\varphi(\widetilde{A})$ as a closed subset of a compact space is normally embedded, and hence there is a metric $\varrho$ on $Q$ with $\varrho(\varphi q(a), \varphi q(b))=d(a, b)$ for $a, b \in A$. Let $X \hookrightarrow \beta X$ be the embedding of $X$ into its Stone-Cech compactification and consider $A$ as a closed subspace of $\beta X$. Since $Q$ is an ANE for compact spaces there is a map $\psi: \beta X \rightarrow Q$ extending $\varphi q$, and then $d^{\prime}(x, y):=\varrho(\psi(x), \psi(y))$ is a metric on $X$ extending $d$.

Proposition 4. For each normal Hausdorff space $X$ and each closed subspace $A$ the natural homomorphism $\lim _{C} H_{*}^{\mathrm{s}}(C, C \cap A ; G) \rightarrow H_{*}^{\mathrm{c}}(X, A ; G)$ is an isomorphism, where the limit is taken over all compact subsets $C$ of $X$.

P r o of. We want to show that for each compact subset $B \subseteq X$ the restriction map $M_{(X, A)} \rightarrow M_{(B, B \cap A)}$ is surjective. Consider a metric $d$ on $B$ with trivial trace on $B \cap A$. Then $d$ may be considered as a metric on $B / B \cap A$, and this space is homeomorphic to the canonical image of $B$ in $X / A$. The assumptions assure that $X / A$ is normal and in particular completely regular, thus by Lemma $8, B / B \cap A$ is normally embedded in 
$X / A$. Therefore $d$ has an extension over $X / A$, i.e. an extension over $X$ with trivial trace on $A$.

Now Theorem 2 permits us to use the directed set $M_{(X, A)}$ as index set of the filtered complexes $\widetilde{\mathbf{C}}_{*}(B, B \cap A ; G)$, and with this understanding we have $\Gamma_{*}^{\mathrm{c}}(X, A ; G)=\bigcup_{B} \Gamma_{*}(B, B \cap A ; G)$.

By means of Proposition 4 the Eilenberg-Steenrod axioms from Section 2 carry over to strong homology with compact supports in the following form:

THEOREM 7. (a)

$$
H_{n}^{\mathrm{c}}\left(S^{0} ; G\right)= \begin{cases}G, & n=0 \\ 0, & n \neq 0\end{cases}
$$

In (b)-(d), $(X, A)$ is a topological pair consisting of a normal Hausdorff space $X$ and a closed subspace $A$ :

(b) There is a natural exact homology sequence

$$
\ldots \rightarrow H_{n}^{\mathrm{c}}(A ; G) \rightarrow H_{n}^{\mathrm{c}}(X ; G) \rightarrow H_{n}^{\mathrm{c}}(X, A ; G) \rightarrow H_{n-1}^{\mathrm{c}}(A ; G) \rightarrow \ldots
$$

(c) The projection map $p:(X \times I, A \times I) \rightarrow(X, A)$ induces isomorphisms of the homology groups $H_{*}^{\mathrm{c}}(p): H_{*}^{\mathrm{c}}(X \times I, A \times I ; G) \rightarrow H_{*}^{\mathrm{c}}(X, A ; G)$.

(d) If $B$ is a closed subspace of $X$ with $A \cap B \neq \emptyset$ and $A \cup B=X$, then the inclusion map $i:(B, A \cap B) \hookrightarrow(X, A)$ induces isomorphisms of homology: $H_{*}^{\mathrm{c}}(i): H_{*}^{\mathrm{c}}(B, A \cap B ; G) \rightarrow H_{*}^{\mathrm{c}}(X, A ; G)$.

In comparison to the homology functor of Section 2 the exactness property of $H_{*}^{\mathrm{c}}$ is improved, because the subspace is no longer required to be normally embedded, but the excision property does not carry over in full strength. The reason is that a compact subset of $X / A$ need not be the image of a compact subset of $X$. However:

Proposition 5. (a) Let $X$ be a paracompact Hausdorff space and $A$ a closed subspace such that $X / A$ is compact. Then $X \backslash A$ is relatively compact in $X$.

(b) For every paracompact space $X$ and every closed, nonempty subspace $A$ the quotient map $p:(X, A) \rightarrow(X / A, *)$ induces isomorphisms $H_{*}^{\mathrm{c}}(p)$ : $H_{*}^{\mathrm{c}}(X, A ; G) \rightarrow H_{*}^{\mathrm{c}}(X / A, * ; G)$.

Pro of. (a) We set $B:=\overline{X \backslash A}$; then by [7, p. 206, Theorem 3.10.21, and p. 306, Theorem 5.1.20] it suffices to show that $B$ is pseudocompact. Now suppose this is false, and let $f: B \rightarrow \mathbb{R}$ be a map and $b_{n}$ a sequence of points with $f\left(b_{n}\right)>n$. By continuity we can find $x_{n} \in B \backslash A$ in some neighborhood of $b_{n}$ with $f\left(x_{n}\right)>n$. The set $C$ formed by all of these points $x_{n}$ cannot

$\left({ }^{1}\right)$ Actually this follows from [21, Corollary 1.2]; we include our proof merely for the sake of completeness. 
have an accumulation point, hence it is a closed discrete set disjoint from $A$. The quotient map $p: X \rightarrow X / A$ maps $C$ homeomorphically onto a closed, discrete subset of $X / A$, which is absurd if $X / A$ is compact.

(b) follows from (a) and Theorem 4.

ExAmple. To show that neither part of the preceding proposition holds for arbitrary spaces $X$ we use a counterexample due to E. Michael [21, Example 3.3]. Let $X$ be the space of all countable ordinals and $A \subseteq X$ the subset of all limit ordinals [7, p. 130, Example 3.1.27]; $X$ is a noncompact, normal Hausdorff space and $A$ is a closed subspace of empty interior. The quotient space $X / A$ is compact, because every neighborhood of $A$ in $X$ has finite complement (hence $X / A$ is homeomorphic to the space $A\left(\aleph_{1}\right)$ in the notation of $[7$, p. 129]).

To show that the quotient map $p:(X, A) \rightarrow(X / A, *)$ need not induce isomorphisms $H_{*}^{\mathrm{c}}(p)$ we use coefficients in a field, say $\mathbb{Z}_{2}$. Strong homology of compact pairs with coefficients in a field coincides with Cech homology, because the inverse limit functor operates exactly on finite-dimensional vector spaces, hence $H_{0}^{\mathrm{c}}\left(X / A, * ; \mathbb{Z}_{2}\right)=H_{0}^{\mathrm{s}}\left(X / A, * ; \mathbb{Z}_{2}\right)=\prod_{\lambda \in X \backslash A} \mathbb{Z}_{2}^{(\lambda)}$, one factor $\mathbb{Z}_{2}$ for each countable nonlimit ordinal. We observe that the number of such ordinals is uncountable. The sets of the form $[0, \lambda]$, where $\lambda$ ranges over the countable ordinals, form a cofinal subset of the set of compact subsets of $X$; this allows us to compute $H_{0}^{\mathrm{c}}\left(X, A ; \mathbb{Z}_{2}\right)$ : It equals the subgroup of $\prod_{\lambda \in X \backslash A} \mathbb{Z}_{2}^{(\lambda)}$ formed by the elements with at most countably many coordinates different from 0 , with $H_{0}^{\mathrm{c}}(p)$ being the inclusion map. Therefore $H_{0}^{\mathrm{c}}(p)$ is not an isomorphism.

Now finally we show that $A$ is normally embedded in $X$, and hence, by [9, Corollary 4], $H_{*}^{\mathrm{c}}$ does not factor over the strong shape category. Let $d$ be a metric on $A$. Then as in [7, Example 3.1.21] one shows that there is an ordinal $\lambda \in A$ such that $d(\mu, \nu)=0$ for all $\mu, \nu \geq \lambda, \mu, \nu \in A$. Since $[0, \lambda]$ is compact and $A$ is closed, the intersection $A \cap[0, \lambda]$ is normally embedded in $[0, \lambda]$, and there is a metric $d^{\prime}$ on $[0, \lambda]$ extending the restriction of $d$. The retraction map $f: X \rightarrow[0, \lambda]$ with $f(\mu)=\mu$ for $\mu \leq \lambda$ and $f(\mu)=\lambda$ for $\mu>\lambda$ is continuous, and $\varrho(\mu, \nu):=d^{\prime}(f(\mu), f(\nu))$ is a metric on $X$ extending $d$. Thus we have proved:

THEOREM 8. Strong homology with compact supports $H_{*}^{\mathrm{c}}$ does not factor over the strong shape category.

THEOREM 9. In ZFC strong homology $H_{*}^{\mathrm{s}}$ does not have compact supports.

This follows from our counterexample simply because $H_{*}^{\mathrm{c}} \neq H_{*}^{\mathrm{s}}$. In [17] Mardešić and Prasolov constructed a different counterexample, but it depended on the continuum hypothesis. 
The last part of this section is devoted to proving isomorphism between strong homology with compact supports and Massey's homology with compact supports. This requires some additional tools, for instance we have to pass from filtered chain complexes to general inverse systems of chain complexes $C_{*}(\lambda)$ and chain maps $h_{\mu}^{\lambda}: C_{*}(\lambda) \rightarrow C_{*}(\mu)$ over some cofinite directed set $\Lambda$. The total complex $\Gamma_{*}$ is defined as in [17, Section 3] using our sign convention of Definition 2. There is an inclusion chain map $i: \lim _{\lambda} C_{*}(\lambda) \hookrightarrow \Gamma_{*}$ mapping a thread of $n$-chains $x_{\lambda} \in C_{n}(\lambda)$ to $\varphi \in \Gamma_{n}$ with

$$
\varphi\left\langle\lambda_{0} \leq \ldots \leq \lambda_{p}\right\rangle:= \begin{cases}x_{\lambda_{0}} & \text { for } p=0 \\ 0 & \text { for } p>0\end{cases}
$$

LEMMA 9. If for each index $\lambda$ the chain map $\lim _{\mu<\lambda} h_{\mu}^{\lambda}: C_{*}(\lambda) \rightarrow$ $\lim _{\mu<\lambda} C_{*}(\mu)$ is surjective and splits as an epimorphism of graded groups, then $i: \lim _{\lambda} C_{*}(\lambda) \hookrightarrow \Gamma_{*}$ is a chain homotopy equivalence.

Proof. (a) First we deal with the special case where the index set $\Lambda$ contains a greatest element $\alpha$. Let $p: \Gamma_{*} \rightarrow C_{*}(\alpha)$ be the evaluation $\operatorname{map} p(\varphi):=\varphi(\alpha)$, and $\varrho: \Gamma_{*} \rightarrow \Gamma_{*+1}$ the chain homotopy $\varrho(\varphi)\left\langle\lambda_{0} \leq \ldots\right.$ $\left.\leq \lambda_{p}\right\rangle:=(-1)^{p+\operatorname{deg} \varphi} \varphi\left\langle\lambda_{0} \leq \ldots \leq \lambda_{p} \leq \alpha\right\rangle$; one checks $p i=\mathrm{id}, \partial \varrho+\varrho \partial=$ $p-$ id and furthermore $\varrho i=0$.

(b) In the proof of the general case we will encounter the following situation: We consider a commutative square of chain complexes as below, and we suppose there exist a chain map $\varphi: X_{*} \rightarrow A_{*}$ with $\varphi i=$ id and a chain homotopy $\varrho: X_{*} \rightarrow X_{*+1}$ with $\partial \varrho+\varrho \partial=i \varphi-$ id and $\varrho i=0$; in addition we assume that $p: E_{*} \rightarrow B_{*}$ is a split epimorphism of graded groups.

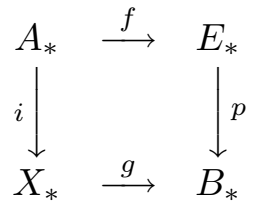

Let $s: B_{*} \rightarrow E_{*}$ be a homomorphism of graded groups with $p s=$ id and set $h:=f \varphi-\partial s g \varrho-s g \varrho \partial: X_{*} \rightarrow E_{*}$. Then $h$ is a chain map fitting commutatively into our diagram.

If two chain maps $h_{0}, h_{1}: X_{*} \rightarrow E_{*}$ with $h_{0} i=h_{1} i$ and a chain homotopy $\tau: X_{*} \rightarrow B_{*+1}, \partial \tau+\tau \partial=p h_{0}-p h_{1}$, with $\tau i=0$ are given, then $\eta:=h_{1} \varrho-$ $h_{0} \varrho+\partial s \tau \varrho-s \tau \varrho \partial: X_{*} \rightarrow E_{*+1}$ is a chain homotopy with $\partial \eta+\eta \partial=h_{0}-h_{1}$, $\eta i=0$ and $p \eta=\tau$.

(c) The general case of the lemma. We consider the subsystems with index set $\{\mu \in \Lambda \mid \mu \leq \lambda\}$ with $\lambda$ fixed and denote the corresponding total complexes by $\Gamma_{*}(\lambda)$; they form an inverse system with limit $\Gamma_{*}$. The 
natural inclusion maps $i_{\lambda}: C_{*}(\lambda) \hookrightarrow \Gamma_{*}(\lambda)$ form a level system of chain maps with limit $i: \lim _{\lambda} C_{*}(\lambda) \hookrightarrow \Gamma_{*}$. Since $\Lambda$ is cofinite we can apply (b) to the diagrams

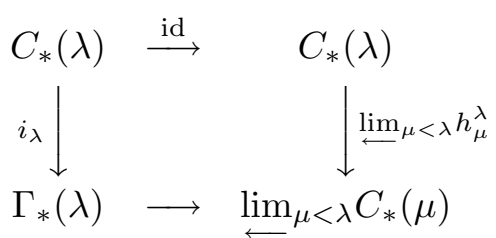

and construct inductively a family of chain maps $f_{\lambda}: \Gamma_{*}(\lambda) \rightarrow C_{*}(\lambda)$ with $f_{\lambda} i_{\lambda}=\mathrm{id}$ and $h_{\mu}^{\lambda} f_{\lambda}=f_{\mu} \gamma_{\mu}^{\lambda}$ for $\mu \leq \lambda$, where $\gamma_{\mu}^{\lambda}: \Gamma_{*}(\lambda) \rightarrow \Gamma_{*}(\mu)$ is the natural projection. These maps determine a limit chain map $f:=\lim _{\lambda} f_{\lambda}$ : $\Gamma_{*} \rightarrow \lim _{\lambda} C_{*}(\lambda)$ with $f i=$ id.

It follows readily from the definitions that $\Gamma_{n}(\lambda)$ equals

$$
\left(\lim _{\mu<\lambda} \Gamma_{n}(\mu)\right) \times \prod_{p \geq 0} \prod_{\lambda_{0} \leq \ldots \leq \lambda_{p-1} \leq \lambda} C_{n+p}\left(\lambda_{0}\right),
$$

so that $\lim _{\mu<\lambda} \gamma_{\mu}^{\lambda}: \Gamma_{*}(\lambda) \rightarrow \lim _{\mu<\lambda} \Gamma_{*}(\mu)$ is a split epimorphism of graded

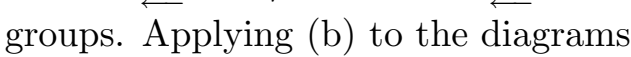

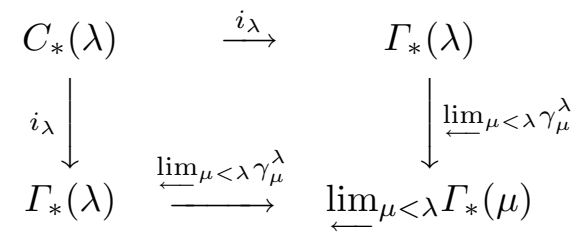

and the maps $i_{\lambda} f_{\lambda}$, id $: \Gamma_{*}(\lambda) \rightarrow \Gamma(\lambda)$ we get a family of chain homotopies $\varrho_{\lambda}: \Gamma_{*}(\lambda) \rightarrow \Gamma_{*+1}(\lambda)$ with $\partial \varrho_{\lambda}+\varrho_{\lambda} \partial=i_{\lambda} f_{\lambda}-\mathrm{id}, \varrho_{\lambda} i_{\lambda}=0$ and $\gamma_{\mu}^{\lambda} \varrho_{\lambda}=\varrho_{\mu} \gamma_{\mu}^{\lambda}$ for $\mu \leq \lambda$. We obtain a limit chain homotopy $\varrho:=\lim _{\lambda} \varrho_{\lambda}: \Gamma_{*} \rightarrow \Gamma_{*+1}$ with $\partial \varrho+\varrho \partial=i f-\mathrm{id}$.

THEOREM 10. On the category of compact Hausdorff spaces and closed subspaces strong homology $H_{*}^{\mathrm{s}}$ is isomorphic to Massey's homology. On the category of normal Hausdorff spaces and closed subspaces $H_{*}^{\mathrm{c}}$ is isomorphic to Massey's homology with compact supports.

COROllary 1. $H_{n}^{\mathrm{c}}=0$ for $n<0$.

Proof of Theorem 10. It is sufficient to consider the compact case and to deal with nonempty spaces $X$ rather than pairs; if we can show that our complex $\widetilde{\Gamma}_{*}(X ; G)$ is naturally equivalent to Massey's chain complex via a sequence of natural chain maps which induce isomorphisms of homology, then the two homology functors will also coincide for pairs and the isomorphism will commute with the boundary operator. 
First we alter the definition of the filtered complex $\widetilde{\mathbf{C}}_{*}(X ; G)$ by replacing oriented simplices $\left\langle x_{0}, \ldots, x_{n}\right\rangle$ of $N_{X}$ by ordered simplices $\left(x_{0}, \ldots, x_{n}\right)$; Theorem 2 ensures that the homology of the total complex remains unaffected. During this proof we will use the old symbol $\widetilde{\mathbf{C}}_{*}(X ; G)$ to denote the new filtered complex.

We set

$$
\Phi^{n}(X):=\left\{\alpha: X^{n+1} \rightarrow \mathbb{Z} \mid \alpha\left(X^{n+1}\right) \text { finite }\right\}
$$

and for $d \in M_{X}$ we denote by $\Phi_{d}^{n}(X) \subseteq \Phi^{n}(X)$ the subgroup of all functions $\alpha: X^{n+1} \rightarrow \mathbb{Z}$ such that $\alpha\left(x_{0}, \ldots, x_{n}\right)=0$ if the set $\left\{x_{0}, \ldots, x_{n}\right\}$ has $d$-diameter at most 1 [20, Ch. $1, \S 1.1]$. The groups

$$
\bar{C}_{n}(d):=\operatorname{Hom}\left(\Phi^{n}(X) / \Phi_{d}^{n}(X), G\right)
$$

constitute a filtered complex $\overline{\mathbf{C}}_{*}(X ; G)$, and the intersection $\bigcap_{d} \bar{C}_{*}(d)$ is precisely Massey's chain complex. For $n=0$ by $\bar{C}_{0}(d)$ we will understand the corresponding reduced complex. There is an obvious chain map $f: \widetilde{\mathbf{C}}_{*}(X ; G) \rightarrow \overline{\mathbf{C}}_{*}(X ; G)$ given by evaluation: $f\left(\left(x_{0}, \ldots, x_{n}\right) \otimes g\right)=\varphi$ with $\varphi(\alpha):=\alpha\left(x_{0}, \ldots, x_{n}\right) g . f$ is injective and has the property $\widetilde{C}_{*}(d)=$ $f^{-1}\left(\bar{C}_{*}(d)\right)$, in particular it is filtration preserving. For each metric $d \in M_{X}$ we can find a finite subset $A \subseteq X$ and a not necessarily continuous function $g: X \rightarrow A$ with $d(x, g(x)) \leq 1 / 4$ for all $x \in X$. The induced vertex map $g: N(2 d) \rightarrow N(d)$ is contiguous to the inclusion map, and applying Lemma 5 we can construct chain homotopies $\widetilde{\varrho}: \widetilde{C}_{*}(2 d) \rightarrow \widetilde{C}_{*+1}(d)$ and $\bar{\varrho}: \bar{C}_{*}(2 d) \rightarrow \bar{C}_{*+1}(d)$ connecting the inclusion map and the chain map induced by $g$ such that the equation $f \widetilde{\varrho}=\bar{\varrho} f$ holds. But since $g$ has finite image one readily sees $g\left(\bar{C}_{*}(2 d)\right) \subseteq \widetilde{C}_{*}(d)$, and now Lemma 6 tells us that the chain map of the total complexes $F: \widetilde{\Gamma}_{*}(X ; G) \rightarrow \bar{\Gamma}_{*}(X ; G)$ determined by $f$ induces isomorphisms of homology.

As explained in the remarks preceding Lemma 9 the inverse limit $\bigcap_{d} \bar{C}_{*}(d)=\lim _{d} \bar{C}_{*}(d)$ may be naturally embedded in the total complex $\bar{\Gamma}_{*}(X ; G)$; and if we can show that the inclusion map induces isomorphisms of homology we are finished. To do this we start by replacing $M_{X}$ by a cofinite index set, taking into account Theorem 2. It follows from Nöbeling's theorem $[20$, p. 88$]$ that the sequence

$$
0 \rightarrow \bar{C}_{n}(d) \rightarrow \operatorname{Hom}\left(\Phi^{n}(X), G\right) \rightarrow \operatorname{Hom}\left(\Phi_{d}^{n}(X), G\right) \rightarrow 0
$$

is split exact; and the collection of them all forms an inverse system of short exact sequences over our new, cofinite index set, giving rise to a short exact sequence of total complexes

$$
0 \rightarrow \bar{\Gamma}_{*}(X ; G) \rightarrow \Gamma_{*}^{\prime} \rightarrow \Gamma_{*}^{\prime \prime} \rightarrow 0
$$


The inverse limit of the sequences (16) is

$$
0 \rightarrow \bigcap_{d} \bar{C}_{*}(d) \rightarrow \operatorname{Hom}\left(\Phi^{*}(X), G\right) \rightarrow \operatorname{Hom}\left(\underline{\lim }_{d} \Phi_{d}^{n}(X), G\right) \rightarrow 0,
$$

and by Nöbeling's theorem this sequence is also exact. The natural inclusions of limit complexes into total complexes provide us with a transformation of the sequence (18) into the sequence (17), whence we derive a transformation of the long exact homology sequences. $\Phi^{*}(X)$ is chain contractible, and hence $\operatorname{Hom}\left(\Phi^{*}(X), G\right)$ and $\Gamma_{*}^{\prime}$ are acyclic, so it remains to show that the natural inclusion

$$
\operatorname{Hom}\left(\lim _{d} \Phi_{d}^{n}(X), G\right)=\lim _{d} \operatorname{Hom}\left(\Phi_{d}^{n}(X), G\right) \hookrightarrow \Gamma_{*}^{\prime \prime}
$$

induces isomorphisms of homology, but since for $d \leq e$ the monomorphism $\Phi_{d}^{n}(X) \hookrightarrow \Phi_{e}^{n}(X)$ splits by Nöbeling's theorem, this follows from Lemma 9.

\section{References}

[1] R. A. Alo and H. L. Shapiro, Normal Topological Spaces, Cambridge Tracts in Math. 65, Cambridge University Press, 1974.

[2] N. A. Berikashvili, Steenrod-Sitnikov homology theories on the category of compact spaces, Soviet Math. Dokl. 22 (1980), 544-547.

[3] -, On the axiomatics of Steenrod-Sitnikov homology theory on the category of compact Hausdorff spaces, Proc. Steklov Inst. Math. 4 (1984), 25-39.

[4] F. W. Cathey and J. Segal, Strong shape theory and resolutions, Topology Appl. 15 (1983), 119-130.

[5] C. H. Dowker, Homotopy groups of relations, Ann. of Math. 56 (1952), 84-95.

[6] D. A. Edwards and H. M. Hastings, Cech and Steenrod Homotopy Theories with Applications to Geometric Topology, Lecture Notes in Math. 542, Springer, 1976.

[7] R. Engelking, General Topology, 2nd ed., Heldermann, Berlin 1989.

[8] B. Günther, Comparison of the coherent pro-homotopy theories of EdwardsHastings, Lisica-Mardešić and Günther, Glas. Mat., to appear.

[9] - , Properties of normal embeddings concerning strong shape theory, II, Tsukuba J. Math., to appear.

[10] Ju. T. Lisica and S. Mardešić, Coherent prohomotopy and strong shape theory, Glas. Mat. 19 (39) (1984), 335-399.

[11] - - - Strong homology of inverse systems of spaces, I, Topology Appl. 19 (1985), $29-43$.

[12] -, - Strong homology of inverse systems of spaces, II, ibid., 45-64.

[13] - - -, Strong homology of inverse systems of spaces, III, ibid. 20 (1985), 29-37.

[14] S. Mardešić, Resolutions of spaces are strong expansions, Publ. Inst. Math. (Beograd) 49 (63) (1991), 179-188.

[15] - Strong expansions and strong shape theory, Topology Appl. 38 (1991), 275-291.

[16] S. Mardešić and Z. Miminoshvili, The relative homeomorphism and the wedge axioms for strong homology, Glas. Mat., to appear. 
[17] S. Mardešić and A. V. Prasolov, Strong homology is not additive, Trans. Amer. Math. Soc. 307 (1988), 725-744.

[18] S. Mardešić and J. Segal, Shape Theory, Math. Library 26, North-Holland, 1982.

[19] S. Mardešić and T. Watanabe, Strong homology and dimension, Topology Appl. 29 (1988), 185-205.

[20] W. S. Massey, Homology and Cohomology Theory, Pure and Appl. Math. 46, Marcel Dekker, 1978.

[21] E. Michael, A note on closed maps and compact sets, Israel J. Math. 2 (1964), 173-176.

[22] Z. Miminoshvili, On the sequences of exact and half-exact homologies of arbitrary spaces, Soobshch. Akad. Nauk Gruzin. SSR 113 (1) (1984), 41-44 (in Russian).

[23] T. Porter, Čech homotopy, I, J. London Math. Soc. (2) 6 (1973), 429-436.

[24] —, Čech homotopy, II, ibid., 662-675.

[25] —, Cech homotopy, III, Bull. London Math. Soc. 6 (1974), 307-311.

[26] E. H. Spanier, Algebraic Topology, McGraw-Hill, 1966.

FACHBEREICH MATHEMATIK

JOHANN WOLFGANG GOETHE-UNIVERSITÄT

ROBERT-MAYER-STRASSE 6-10

6000 FRANKFURT, GERMANY

Current address:

HELPERTSEESTRASSE 21

D-6052 MÜHLHEIM, GERMANY 